\title{
Nuevos registros de arañas (Araneae: Arachnida) para la Región de Arica y Parinacota, Norte de Chile
}

\author{
New records of spiders (Araneae: Arachnida) for the Arica and Parinacota Region, \\ Northern Chile
}

Andrés Taucare-Ríos ${ }^{1 *}$

\section{RESUMEN}

A partir de arañas adultas colectadas en el valle de Azapa se actualiza el número de especies presentes en la Región de Arica y Parinacota. Se entregan datos ecológicos y distribucionales de las especies encontradas y una breve diagnosis para poder identificarlas. Palabras clave: arañas, ecología, valle de Azapa, Norte de Chile.

\begin{abstract}
From adult spiders collected in the Azapa Valley the number of species present in the region of Arica and Parinacota is updated. Ecological and distributional data of the species found are given and a brief diagnosis for identification is provided.

Key words: spiders, ecology, azapa valley, northern Chile.
\end{abstract}

Las arañas (Arachnida: Araneae) comprenden un grupo faunístico diverso y ampliamente distribuido en todos los ecosistemas terrestres, colonizando incluso ambientes dulceacuícolas (Turnbull, 1973). El orden cuenta con alrededor de 45.000 especies descritas (Platnick, 2014) y está considerado como uno de los grupos entomófagos más abundantes en la naturaleza (Nyffeler et al., 1994). Se ha propuesto incluso (Clausen, 1986) que las arañas son organismos ideales para sondeos biológicos, principalmente los enfocados a las descripciones de biotopos y a los seguimientos de agentes contaminantes. De esta manera, estos artrópodos conforman un grupo muy importante para la ecología, ya que pueden ser utilizados como agentes indicadores de cambios ambientales (Rubio et al., 2007).

En la Región de Arica y Parinacota los estudios relacionados con la araneofauna son más bien escasos. Actualmente para la región se conocen 26 especies (Taucare-Ríos \& Sielfeld, 2013) en sus diferentes ambientes, que van desde la costa, pasando por el desierto absoluto y sus bofedales altoandinos. Sin embargo aún no existe certeza absoluta de las especies presentes y mucho menos datos vinculados con su distribución e historia natural.
El objetivo de este trabajo es actualizar la composición faunística de las arañas presentes en la Región de Arica y Parinacota a partir de una serie de colectas realizadas durante el 2013 en el valle de Azapa y alrededores. Se entregan datos ecológicos y de distribución para las especies que constituyen nuevos registros. Se ha elegido este lugar de estudio debido al gran desconocimiento que se tiene respecto de las arañas en zonas desérticas, sumado a la relevancia que tiene para la comunidad conocer la fauna de arañas presentes en la región. De esta manera, este trabajo constituye un real aporte al conocimiento de la araneofauna de zonas áridas, estableciendo las bases para futuros estudios ecológicos y taxonómicos en la Región de Arica y Parinacota.

Las arañas fueron identificadas a nivel de especie siguiendo trabajos taxonómicos específicos (Levi, 1967, 2004; Taucare-Ríos, 2011, 2012, 2013a,b; Taucare-Ríos \& Sielfeld, 2013) y fijadas en etanol al 95\%. Las técnicas de recolecta utilizadas fueron el apaleo de follaje y capturas manuales directas. El material biológico se encuentra depositado en el Museo Nacional de Historia Natural (MNHNCL, Curador, Mario Elgueta) de Santiago de Chile.

\footnotetext{
1 Centro de Investigación en Medio Ambiente (CENIMA), Universidad Arturo Prat, Casilla 121, Iquique, Chile.

* Autor para correspondencia: and.taucare26@gmail.com
} 
Las familias de arañas se clasificaron en dos gremios ecológicos basados en la estrategia de forrajeo adoptada por el depredador en relación con la presa (sensu Gerscth, 1979): las arañas cazadoras (i.e., arañas que se alimentan sin el uso de una red de captura) y las arañas constructoras de telas (i.e., que son aquellas que sí construyen red de captura para cazar a sus presas). Las abreviaturas utilizadas al lado del nombre científico de cada especie son las siguientes: $\mathrm{AC}=$ Arañas errantes cazadoras; $\mathrm{CT}$ $=$ Arañas constructoras de telas; $\mathrm{P}=$ Peligrosa $; \mathrm{NP}$ $=$ No peligrosa.

\section{Listado de Especies}

\section{Familia Salticidae}

\section{Hasarius adansoni (Audouin, 1826) (AC) (NP)}

Registros previos: Previamente ha sido reportado para Pisagua e Iquique en la Región de Tarapacá (Taucare-Ríos, 2013b).

Nuevos registros e historial natural: Se ha encontrado una hembra adulta de esta especie para la localidad de San Miguel de Azapa, ampliando su distribución septentrional en Chile. Es común ver estas arañas en patios y antejardines de las casas (Figura 1), donde buscan presas (usualmente grillos y moscas) para cazar.

\section{Familia Theridiidae}

\section{Latrodectus geometricus C. L. Koch, 1841 (CT) (P)}

Registros previos: Previamente ha sido encontrada en áreas urbanizadas de Iquique y Azapa (Taucare-Ríos, 2011; Taucare-Ríos \& Sielfeld, 2013).

Nuevos registros e historia natural: Se amplía su distribución hacia la ciudad de Arica a base de una hembra colectada recientemente. Estas arañas tejen sus telas irregulares y difusas para atrapar a sus presas, haciendo sus nidos en el exterior de las casas, parques y postes de luz de la ciudad (Figura 2). Esta araña es una especie invasora y ha sido introducida accidentalmente en el norte de Chile por la actividad antrópica (Taucare-Ríos, 2011). Si bien es una especie tímida y de mediano tamaño es considerada como una araña de importancia médica (Taucare-Ríos, 2013).

\section{Familia Filistatidae}

Kukulcania brevipes (Keyserling, 1883) (AC) (NP)

Registros previos: Esta especie es nativa de Perú y fue recientemente reportada para la localidad de Arica a partir de una hembra adulta colectada en ambientes urbanos (Brescovit \& Santos, 2013).

Nuevos registros e historia natural: A partir de 4 hembras adultas se amplía la distribución de esta especie para la localidad de San Miguel de Azapa. Esta araña es de un color marrón oscuro (Figura 3) muy similar a Loxosceles laeta, con la cual podría ser confundida, no obstante, pese a su parecido es completamente inofensiva. Esta especie teje una tela en forma de embudo o tubo en las viviendas de la localidad de Azapa, siendo su tela considerablemente distinta de la tela algodonosa y desordenada de la araña de los rincones.

\section{Familia Scytodidae}

\section{Scytodes univitatta (Simon, 1882) (AC) (NP)}

Registros previos: Esta especie ha sido citada para Poconchile, Pisagua, Iquique y Alto Hospicio (Taucare-Ríos, 2013), siendo más abundante en la región de Tarapacá.

Nuevos registros e historia natural: Se amplía su distribución a San Miguel de Azapa a base de una hembra encontrada bajo piedras en un antejardín. Es una especie sinantrópica inofensiva, asociada principalmente a áreas urbanizadas (Figura 4), su dieta se basa principalmente en otras arañas, lo que la convierte en una araña esencialmente aracnofágica.

Consideraciones. Con este trabajo se documenta la ampliación de distribución de cuatro especies para la región de Arica y Parinacota, donde una de ellas constituye el primer registro para esta zona (Hasarius adansoni). De estos nuevos registros tres de ellos corresponden a arañas cazadoras errantes y una de ellas al gremio ecológico de arañas constructoras de telas. Las especies Hasarius adansoni, Latrodectus geometricus y Scytodes univitatta son especies exóticas introducidas en el país (Taucare-Ríos \& Sielfeld, 2013) , mientras que Kukulcania brevipes corresponde a una especie nativa de Perú y norte de Chile (Brescovit \& Santos 2013), siendo muy abundante en las casas de San Miguel de Azapa y 

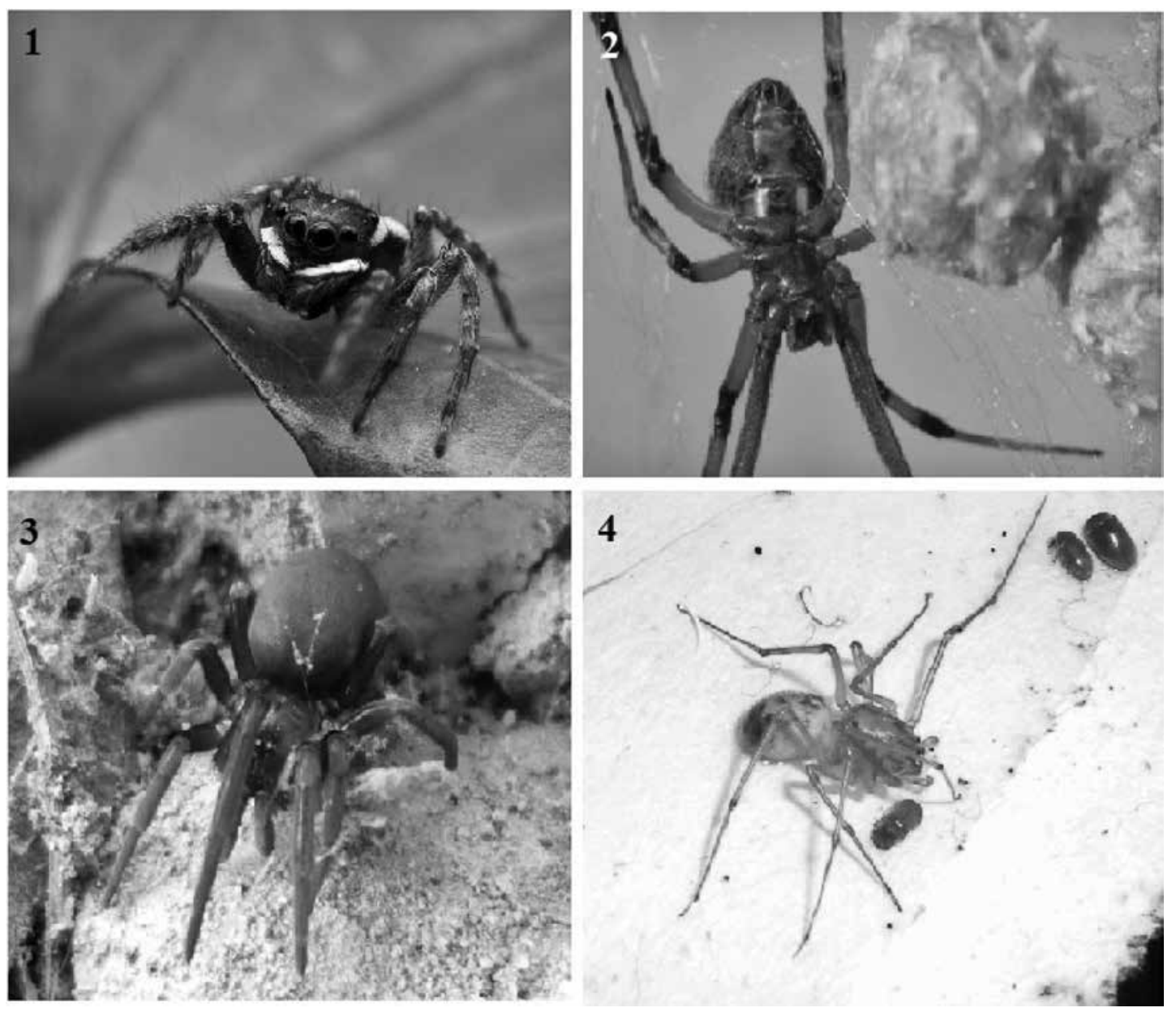

Figuras 1-4. 1. Hembra de Hasarius adansoni (Salticidae); 2. Hembra de Latrodectus geometricus (Theridiidae) y sus cocoones; 3. Hembra de Kukulcania brevipes (Filistatidae); 4. Macho de Scytodes univitatta (Scytodidae).

alrededores. Se sabe que la fauna de arañas de esta región es una de las menos estudiadas en el país y por ese motivo estos nuevos registros contribuyen a un mejor conocimiento de la araneofauna local, no obstante, existen ambientes que no han sido suficientemente explorados por lo que habría que esperar que el número de especies en la región aumentara. En este contexto, también es imperioso hacer extensivos estos estudios al resto de las zonas áridas chilenas, donde los estudios aracnológicos aún son considerablemente exiguos.

\section{Agradecimientos}

Se agradece a la beca CONICYT N 21130014 para estudios de Doctorados Nacionales en Chile.

\section{Literatura Citada}

Brescovit, A.; Santos, A.

2013. The spider genus Kukulcania in South America (Araneae: Filistatidae): a redescription of $K$. brevipes (Keyserling) and new records of K. hibernalis (Hentz). Zootaxa 3734 (3): 301-316.

Clausen, $\mathrm{S}$.

1986. The use of spiders (Araneae) as ecological indicators. Bulletin from Brasilian Arachnology Society, 7: 83-86.
Gertsch W.J.

1979. American Spiders, 2nd edition. New York: Van Nostrand Reinhold. 196 p.

Nyffeler, M.W.; W.L. Sterling; D. Dean

1994. How spiders make a living. Environmental Entomology, 23: $1357-1367$. 
Platnick, N.I.

2014. The World Spider Catalog, V. 14.5. American Museum of Natural History. URL: http://research.amnh.org/entomology/ spiders/catalog/ Consultado: Abril 19, 2014.

Rubio, G.D.; I. Minoli; L.N. Piacentini

2007. Patrones de abundancia de cinco especies de arañas lobo (Araneae: Lycosidae) en dos ambientes del Parque Nacional Mburucuyá, Corrientes, Argentina. Brenesia, 67, 59-67.

Taucare-Ríos, A.

2011. Primer registro de la viuda marrón, Latrodectus geometricus Koch, 1841 (Araneae: Theridiidae) en el norte de Chile. Revista Chilena de Entomología 36: 39-42.
Taucare-Ríos, A.

2013a. El género de arañas Scytodes Latreille, 1804 (Araneae: Scytodidae) en Chile: diversidad y distribución. Revista Chilena de Historia Natural 86: 103-105.

Taucare-Ríos, A.

2013b. Primeros registros de la araña saltarina Hasarius adansoni (Auodouin, 1826) (Araneae: Salticidae) en Chile. Idesia 31 (2): 103-105.

Taucare-Ríos, A.; Sielfeld, W.

2013. Arañas (Arachnida: Araneae) del extremo norte de Chile. Boletín del Museo Nacional de Historia Natural 62: 7-27.

Turnbull, A.

1973. Ecology of the true spiders. Annual Review of Entomology. 18: 305-348. 